\title{
A rare presentation of Pediatric Autoimmune Neuro-psychiatric Disorder Associated with Group A Streptococcal infections (PANDAS)
}

\author{
Sucharita Datta, MD ${ }^{1}$ Saumyen De, $\mathrm{MD}^{2}$, Anand Prasad ${ }^{3}$, Ujjal Mondal ${ }^{4}$, \\ ${ }^{1}$ Associate Professor, ${ }^{2}$ Assistant Professor, ${ }^{3,4}$ Post Graduate Trainee, Department of Pediatrics, Nilratan Sircar \\ Medical College, West Bengal University of Health Sciences, India
}

\begin{abstract}
A 9 year old girl presented to us with a history of sudden onset left sided hemiparesis. Neurological examination revealed an upper motor neuron type of left facial nerve palsy, episodes of dystonia involving left upper and lower limbs and an extensor plantar response on the left side. The girl showed several adventitious movements such as choreiform movements involving the whole of the left upper extremity and the distal left lower extremity. Additionally, facial tics causing a grimacing facies were seen off and on. Interestingly, a mitral regurgitation murmur was discovered on routine clinical examinations and confirmed by echocardiography. With this background and a past history of acute tonsillitis 3 years back, we got two Anti-Streptolysin O (ASO) titres done 3 weeks apart which were markedly elevated and showed a rising titre; $406 \mathrm{IU} / \mathrm{L}$ and $815 \mathrm{IU} / \mathrm{L}$ respectively. The girl was started on oral prednisolone, oral penicillin and oral haloperidol and she responded dramatically within 5 days. Stroke syndrome, as a cause of the acute hemiparesis, was ruled out by neuroimaging (MR-angiography). The clinical profile of our case fulfilled all the diagnostic criteria for " Pediatric Autoimmune Neuro Psychiatric Disorder Associated with Streptococcal infections" (PANDAS). But here, we are reporting not just another case of PANDAS, but one with a unique difference: the association of complete hemiparesis with PANDAS.
\end{abstract}

Keywords - ASO titre, choreiform movements, hemiparesis, PANDAS, Tics

\section{INTRODUCTION}

The term PANDAS proposed by Swedo et al ${ }^{1}$ in 1998 was characterized by the presence of childhoodonset obsessive compulsive disorder (OCD) and/ or tic disorder which have their onset and subsequent exacerbations temporally related to group A beta haemolytic Streptococcal infection (GABHS) . Despite more than a decade of studying PANDAS, many controversies remain regarding validation of diagnostic criteria, inconclusive evidence of autoimmunity, weak evidence of genetic predisposition and doubts about temporal relation to GABHS ${ }^{2}$.

The concept of PANDAS evolved out of some similarities with Sydenham's chorea (SC)- a neurologic manifestation of rheumatic fever ( RF), based on the hypothesis of an autoimmune disorder triggered by a GABHS infection, wherein a molecular mimicry process might occur whereby antibodies directed against bacterial antigens cross-react with brain targets ${ }^{3}$.

We are reporting here a post-streptococcal phenomenon in a 9 year old girl satisfying all the 5 diagnostic criteria of "PANDAS" with a never reported association of " complete hemiparesis".

\section{CASE REPORT}

A 9 year old girl presented to us with weakness of the left upper and lower limbs and deviation of the angle of the mouth to the right since 3 weeks back. On detailed history-taking, it was revealed that one morning on waking up, the girl suffered frequent falls while walking. To the utter surprise of her mother, by late afternoon, the girl was just unable to walk and was confined to her bed. The mother also noticed her facial asymmetry and abnormal facial contour soon after. There was no history of fever, convulsion, vomiting, headache or alteration of sensorium either preceding or following the onset of her neurologic symptoms. There was no contact history of tuberculosis in the family.

On general examination, the girl was found to be alert, conscious and co-operative. Pallor, cyanosis, clubbing and jaundice were absent. Pulse rate was $88 / \mathrm{min}$ and was of normal character. Blood pressure was within normal limits in both upper and lower limbs. Temperature was normal. Lymph nodes were not palpable and there was no evidence of raised jugular venous pressure.

On neurological examination, the girl was found to be well-oriented. Cranium and spine showed no abnormalities. Speech was normal. Other than an upper motor neuron type of facial nerve palsy of the left side, rest of the cranial nerve examination proved normal. 
The left upper and lower limbs showed intermittent bouts of dystonia on passive movement, with normal tone in between. Power was also reduced in both upper and lower limbs on the left side with the upper limb showing greater weakness (Muscle power 2/5) than the lower limb(Muscle power 3/5). Deep tendon reflexes (DTR) were exaggerated on left side with an extensor left sided plantar response. The tone, power, DTR and plantar response were all normal on the right side. The girl showed no features of sensory, autonomic or cerebellar dysfunction. By the time that the girl was admitted ,( day 21 of her illness) the girl could sit-up from supine position and stand on her own without support - albeit with a broad based stance. She could walk a few steps with a hemiplegic gait.

During the course of the neurologic examination, she used to frequently blink her eyes. Involuntary dyskinetic movements around the mouth giving her a grimacing facial appearance occurred off and on but were irregular and for a brief period --- evidently motor tics. On enquiring about the tics, her mother informed that though present earlier, she noticed an aggravation of these movements soon after the left sided weakness of limbs.

We made an interesting observation while eliciting the DTRs' and plantar reflex in the initial 5 to 7 days after admission both in the supine and sitting posture (with lower limbs hanging free from the edge of the bed). There were sudden repetitive irregularly timed involuntary flexion movements of the left hand at the wrist with quick twitches of the fingers. Similar movements were elicited in the left lower leg and ankle. Bizarre stereotyped undulating movements involving the left shoulder elbow and wrist, causing the left arm to swing obliquely to the right side, brushing against her nose in the process-were seen.

During routine clinical examination, we found evidence of cardiomegaly with apex beat on the left fifth intercostal space, $2.5 \mathrm{~cm}$ outside the midclavicular line. An unexpected finding was that of a pansystolic murmur in the mitral area (Grade3/6). Liver spleen and lymph nodes were not palpable. Examination findings were normal for other systems.

Investigations: The complete blood count ( TLC-8400/ cu mm;DLC- $\mathrm{N}_{50} \quad \mathrm{~L}_{40} \mathrm{M}_{2} \mathrm{E}_{8}$ Platelet count-2.4 lacs/cu mm) was normal except for a high ESR of $64 \mathrm{~mm}$ in the first hour. Blood urea $-17 \mathrm{mg} / \mathrm{dL}$, serum creatinine- $0.7 \mathrm{mg} / \mathrm{dL}$. Serum sodium and potassium were within normal limits. Mantoux test was negative. Test for ANA was nonreactive. ECG tracing was normal with normal PR interval. The chest X-ray revealed cardiomegaly. A plain CT scan of brain was within normal limits. MRI brain was done to disclose any abnormality perhaps missed on CT scan. This too proved to be normal. Echocardiography revealed severe mitral regurgitation, thereby corroborating our clinical finding. Other valves were morphologoically normal and no vegetations were seen in any of the valves.

Our initial suspicion regarding whether this case could be a case of childhood stroke syndrome due to embolism from rheumatic vegetations was dispelled from the echocardiographic findings and the MRI scans.

Nevertheless, keeping in mind that vasculitis could lead to a stroke syndrome, we did an MR angiography which again proved to be normal.

In the light of the girl's present clinical scenario and history of acute tonsillitis 3 years earlier and an ASO test done 2 weeks prior to admission showing an elevated titre of 406 IU/L, we repeated the ASO test one week post-admission and observed a two-fold rise in titre (815 IU/L).

Rheumatic fever (RF) and/ or Sydenham's chorea (SC) were nevertheless ruled out as will be evident later in the discussion.

In an attempt to rule out Neuro-Wilson, a slit lamp examination for Kayser-Fleischer ( KF) ring was done and was found to be normal. The serum ceruloplasmin was also normal ( $38 \mathrm{mg} / \mathrm{dL}$ ).

After extensive review of literature the controversial 'PANDAS' attracted our attention and the diagnosis established as per diagnostic criteria of PANDAS. Accordingly, a course of oral Prednisolone, oral Penicillin and Haloperidol were given with dramatic response within 5 days.

\section{Discussion}

This nine-year old girl presented to us with complete hemiparesis of the left side, facial asymmetry and deviation of the angle of the mouth to the right. Due to the sudden onset, the possibility of stroke was considered. In the course of the neurological examination, the frequent eye blinking and dyskinetic movements around the mouth causing a stereotypic facial grimace were noticed which on enquiring, the mother stated that the abnormal facial movements which were present previously too, aggravated almost concurrently with the hemiparesis. While eliciting the knee jerks in the sitting posture on the edge of the bed with the legs hanging loose, there were abrupt flexion movements at the left wrist and ankle joint, the movements were irregularly timed with variable frequency. There were also some haphazard movements initiating from the left shoulder joints progressing to involve the left elbow, arm and the wrist in undulating writhing movements, causing the left arm to swing towards the right with the left hand brushing against her nose. On asking the girl's mother about these choreiform movements, she categorically said that she marked such movements whenever a neurological examination was in progress and not otherwise. This observation corroborates the definition by 
Murphy et al ${ }^{4}$ of "choreiform movements" as the movements elicited by a clinician exclusively upon neurological examination ( Stressed posture). Murphy on the other hand defines 'chorea' as unsolicited spontaneous movements observe in a child at rest ${ }^{4}$.

During routine clinical examination of the other systems, a pansystolic murmur was discovered in the mitral area, suggesting mitral regurgitation which was confirmed by echocardiography as severe mitral regurgitation. The other valves were morphologically normal with no vegetation seen in any of the valves. The pericardium was normal. Childhood stroke syndrome due to embolism from vegetation as a cause of sudden onset hemiplegia was ruled out by an otherwise normal echo report other than severe MR, and normal neuroimaging ( normal CT, MRI and MR angio).Vasculitis was also ruled out by nonreactive ANA and normal neuroimaging.

As occasionally SC may be the presenting symptom of $\mathrm{RF}^{5,6}, \mathrm{SC}$ was taken into consideration in spite of the elevated and rising ASO titre.Frank chorea (rapid, irregular and nonstereotypic jerks that are continuous while the patient is awake but improve with sleep) ${ }^{5,7}$ and hypotonia suggest Sydenham chorea ${ }^{5,6}$. But our patient showed subtle choreiform movements which were elicited through stressed postures during neurological examination but were not present at rest and occurred in a stereotyped pattern. The girl also had normal tone in all four limbs except occasional dystonia on the left side during examination unlike 'hypotonia' in SC.

According to the Revised ( 1992) Jones criteria for the diagnosis of rheumatic fever (RF), ' 2 major criteria or 1 major and 2 minor criteria plus proof of recent GABHS infection' are to be fulfilled. But our case , with carditis as evidenced by the auscultatory finding of MR and Doppler echocardiographic confirmation of MR and an elevated ESR with an elevated and rising trend of ASO titre (i.e, 1 major, 1 minor criteria plus proof of recent GABHS infection) did not satisfy the diagnostic criteria of RF too.

A normal serum ceruloplasmin and absence of KF ring on slit lamp examination of eye negated the possibility of Neuro-Wilson.

It was at this point, that the concept of PANDAS evolved in our mind as it has been stressed by certain researchers that if PANDAS syndrome represents part of the RF complex spectrum, there should be an increased prevalence of rheumatic heart disease in these patients since it is the most severe autoimmune sequel of GABHS infections ${ }^{8}$.

In one study, out of 66 children with tic disorder, 48 children (72\%) had streptococcal exposure out of which 26 children $(54.3 \%)$ had a mitral regurgitation ${ }^{9}$. In the same study it was observed that in children with tic disorder showing mitral regurgitation, a significantly higher rate of subjects $(26.9 \%$ vs $4.5 \%$; $p$ value $<0.05)$ showed choreiform movements in comparison with tic patients without cardiac abnormalities. Moreover, 4/6 children $(66 \%)$ meeting the diagnostic criteria of PANDAS showed MR.

Our case fulfilled all the five diagnostic criteria of PANDAS viz:i) the presence of tic disorder in the form of repeated eye blinking and orofacial dyskinesia with grimacing, ii) prepubertal age of onset at 9 years ( usually between 3 and 12 years of age), iii) Abrupt symptom onset ( sudden exacerbation of tics),iv)Temporal relation between group A streptococcal (GAS) infection and onset of symptoms and v) presence of neurologic abnormalities, such as motor hyperactivity, choreiform movements ( elicited through stressed posture but not present at rest), or tics during exacerbations ${ }^{10,11,12}$. The unsual association of hemiparesis with PANDAS has not been found after extensive search of literature. However in a published symposium on neurology ${ }^{13}$, on the differential diagnosis, investigations and management of a child with suspected stroke, Post streptococcl hemiparesis, dystonia and chorea associated with throat infections, positive ASO titre and MRI showing signal change not typical for ischemia and penicillin for treatment as for typical Sydenham's Chorea ${ }^{13}$ has been mentioned. The presence of severe MR in our case has also been amply corroborated in the foregoing discussion.

To conclude, GABHS infection should be considered in any patient presenting with tics or OCD . Conventional drugs for OCD or tics are the mainstay of management of PANDAS. Antibiotics such as Penicillins/ cephalosporin are recommended for children with positive throat or skin culture for GABHS or rapid antigen detection test. Immunomodulators such as steroids, plasma exchange or IVIG can be used in the research setting ${ }^{12}$. Cardiac involvement is common in PANDAS.

Our case of PANDAS is unique due to its association with hemiparesis---- hitherto not reported.

\section{REFERENCES}

[1]. Swedo SE, Leonard HL, Garvey M, et al.: Pediatric autoimmune neuropsychiatric disorders associated with streptococcal infections:clinical description of the first 50 cases. Am J Psychiatry 1998,155:264-271.

[2]. de Oliveira SK, Pelajo CF.Pediatric Autoimmune Neuropsychiatric Disorders Associated with Streptococcal Infection (PANDAS): a Controversial Diagnosis. Curr Infect Dis Rep. 2010, 12(2):103-9

[3]. CunninghamMW: Pathogenesis of group A streptococcal infections. Clin Microbiol Rev 2000, 13:470-511.

[4]. Murphy TK, Snider LA, Mutch PJ, et al.: Relationship of movements and behaviors to group A Streptococcus infections in elementary school children. Biol Psychiatry 2007, 61:279-284. This article describes a community-based longitudinal study that enrolled 693 children and determined prevalence of group A streptococcal infections, tic behaviors, and choreiform movements.

[5]. Zomorrodi A, Wald ER. Sydenham's Chorea in Western Pennsylvania . Pediatriatrics. 2006; 117(4):675-679 
[6]. Swedo SE,Leonard HL, Shapiro MB. Sydenham's Chorea:Physical and Psychological Symptoms of St Vitus Dance. Pediatrics.1993; 91(4): 706-713

[7]. Aron AM, Freeman JM, Carter S. The Natural History Of Sydenham's Chorea. Review Of The Literature And Long-Term Evaluation With Emphasis On Cardiac Sequelae. Am J Med 1965; 38:83.

[8]. Kaplan EL. PANDAS? or PAND? or both? or neither? Assessing a (possible?) temporal or pathogenetic ralationship with the group A “streptococcal diseases complex". Contemp Pediatr 2000;17:81-96.

[9]. F Cardona, F Ventriglia, O Cipolla, A Romano, R Creti, G Orefici. A post-streptococcal pathogenesis in children with tic disorders is suggested by a color Doppler echocardiographic study. European Journal of Paediatric Neurology 2007; 11( 5): 270-276

[10]. Swedo SE, Leonard HL, Garvey M, et al. Pediatric autoimmune neuropsychiatric disorders associated with streptococcal infections: clinical description of the first 50 cases. Am J Psychiatry 1998; 155:264.

[11]. Allen AJ, Leonard HL, Swedo SE. Case study: a new infection-triggered, autoimmune subtype of pediatric OCD and Tourette's syndrome. J Am Acad Child Adolesc Psychiatry 1995; 34:307.

[12]. Swedo SE, Leonard HL, Rapoport JL. The pediatric autoimmune neuropsychiatric disorders associated with streptococcal infection (PANDAS) subgroup: separating fact from fiction. Pediatrics 2004; 113:907.

[13]. Kirkham F. Investigation and management of childhood stroke: Paediatrics And Childhealth. 2010 ; 20( 9):428-438 